\title{
La investigación en la disciplina de la orientación: procesos de formación desde la percepción estudiantil
}

\author{
Research in the Discipline of Counseling: Educative Processes From Students' Perception
}

\section{A investigação na disciplina da orientação: Processos de formação a partir da percepção do grupo de estudantes}

\author{
Cindy Vanessa Artavia-Aguilar \\ Universidad Nacional \\ Centro de Investigación y Docencia en Educación \\ Heredia, Costa Rica \\ cindy.artavia.aguilar@una.cr \\ http://orcid.org/0000-0002-2179-6808 \\ Luis Roberto Campos-Hernández \\ Universidad Nacional \\ Centro de Investigación y Docencia en Educación \\ Heredia, Costa Rica \\ robertocamp04@yahoo.com \\ http://orcid.org/0000-0001-9459-1662
}

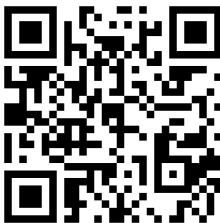

Recibido • Received • Recebido: 28 / 08 / 2018

Corregido • Revised • Revisado: 27 / 02 / 2020

Aceptado • Accepted • Aprovado: 01 / 04 / 2020

\begin{abstract}
Resumen: Este artículo surge ante la necesidad de sistematizar las experiencias vividas, reflexiones y discusiones en torno a los procesos de formación investigativa en la carrera de Orientación de la Universidad Nacional, desde la perspectiva estudiantil. Con este fin, se plantea el objetivo de indagar los requerimientos laborales en el área de investigación, así como si la estrategia pedagógica empleada en esta materia es aplicable al quehacer profesional. El estudio es cuantitativo con alcance exploratorio; en este, se les solicitó a 15 estudiantes del curso Metodología de investigación de la licenciatura en Orientación en los años: 2014, 2015, 2016 y 2017 que realizaran un análisis de fortalezas, oportunidades, debilidades y amenazas (FODA) a este eje curricular, para recoger información en la categoría "percepción de la formación académica en investigación", y completaran un cuestionario con preguntas abiertas y semi-estructuradas, en las categorías "características de una persona orientadora-investigadora" y "aplicabilidad de la investigación en el quehacer profesional". Dentro de las principales conclusiones, destaca la trascendencia de la investigación en la vida profesional de la persona Orientadora, y la importancia de la integralidad de estos procesos en todo el quehacer profesional y de formación académica.
\end{abstract}

Palabras claves: Investigación; orientación; formación académica; educación universitaria; percepción estudiantil. 
http://doi.org/10.15359/ree.24-2.13

ROR: https://ror.org/01t466c14 Universidad Nacional, Costa Rica

http://www.una.ac.cr/educare

educare@una.cr

Abstract: Writing this article responds to the necessity to systematize the experiences, reflections, and discussions about the process of research training from the student's perspective in the major of Counseling program at the National University (UNA). The aim is to explore the work requirements in the research field and to determine the applicability of pedagogical strategies in professional daily work. This research has an exploratory quantitative design, in which 15 students of the course Research Methodology, from the bachelor's degree program in Counseling in 2014, 2015, 2016 y 2017, carried out an analysis of the strengths, weaknesses, opportunities, and threats (SWOT) to this curricular area to pick up information in the category "Perception of the Academic Training in Research." Besides, they completed an open-questioned and semi-structured questionnaire regarding the characteristics that the person from the Counseling major needs to become a researcher, as well as the applicability of research in the everyday professional work. One of the main conclusions is the necessity of highlighting the significance of research in the guidance counselors' professional area, and the importance that integrality of processes has around the professional endeavor and the academic training.

Keywords: Research; orientation; academic formation; university education.

Resumo: Este artigo decorre da necessidade de sistematizar as experiências, reflexões e discussões sobre os processos de formação em pesquisa na carreira de Orientação, da Universidade Nacional, desde a perspectiva das pessoas estudantes. Para tanto, propõe-se o objetivo de investigar as exigências trabalhistas na área de pesquisa, bem como se a estratégia pedagógica utilizada nessa área é aplicável ao trabalho profissional. O estudo é quantitativo com transfundo exploratório. Nesse sentido, 15 alunos do curso de Metodologia da Pesquisa do Bacharelado em Orientação nos anos: 2014, 2015, 2016 e 2017 foram convidados a realizar uma análise de forças, oportunidades, fraquezas e ameaças (FODA, segundo as siglas do espanhol) a este eixo curricular, coletar informações na categoria "percepção da formação acadêmica em pesquisa" e preencher um questionário com perguntas abertas e semiestruturadas, nas categorias "características de um orientador-pesquisador" e "aplicabilidade da pesquisa no trabalho profissional". Entre as principais conclusões, destaca-se a importância da pesquisa na vida profissional da pessoa orientadora e a importância da integralidade desses processos em todo o trabalho profissional e formação acadêmica.

Palavras-chave: Pesquisa; orientação; formação acadêmica; educação universitária.

\section{Introducción}

En una sociedad cada vez más cambiante y compleja, donde los adelantos científicos establecen las pautas de desarrollo y cuyos hallazgos son más fácilmente accesibles a la población en general, la disciplina de Orientación se enfrenta a una gran diversidad de contextos y retos sociales que influyen en el quehacer profesional e implican reflexiones constantes sobre la praxis y teorización de los fundamentos disciplinares.

Uno de los ejes curriculares en Orientación es la investigación, la cual se integra a la vida estudiantil desde el primer año de carrera, en los objetivos propios de esta disciplina, las prácticas profesionales, los trabajos finales de graduación, los cursos de la carrera y trabajos de 
aula, hasta finalizar el plan de estudios de bachillerato y licenciatura. El trabajo investigativo debe integrarse, a su vez, al quehacer docente y programas de estudio, así como, realimentar la malla curricular de la carrera.

La investigación se ha visualizado durante mucho tiempo como un aspecto a evaluar dentro trabajos académicos y como elemento conclusivo en la licenciatura en Orientación; además, se han realizado esfuerzos por integrar este eje al plan de estudios, de manera tal que constituya un proceso continuo en el tiempo de formación y posterior al egreso de la carrera.

Se consideró importante analizar cómo el colectivo estudiantil percibe estos procesos, y los posibles cambios de criterios relacionados con metodología de investigación y sus implicaciones en toda la carrera, en un periodo de cuatro años. Lo anterior con la finalidad de integrar aportes del estudiantado y diferentes académicos en los cursos que abordan este contenido en licenciatura, así como la experiencia de los docentes de curso, para atender las nuevas demandas socio-culturales, pedagógicas y epistémicas en esta área. Con el presente artículo, se pretende no solo dejar evidencia de los procesos que se llevan a cabo, sino contribuir a los cambios a nivel curricular, pedagógico y de acercamiento a la docencia.

\section{Referente conceptual}

\section{El papel de la investigación en la disciplina de Orientación}

El trabajo investigativo es un pilar fundamental en la formación académica y el ejercicio de las personas profesionales en Orientación. En la Universidad Nacional, este se visualiza dentro del plan de estudios de la carrera como un eje curricular y se asume "como proceso intencional, sistemático y sistematizable" (Universidad Nacional, Centro de Investigación y Docenciaen Educación, División de Educación para el Trabajo, 2016, p. 26), el cual se caracteriza por ser continuo y dinámico en construcción de conocimiento. Se hace presente como eje curricular en varios cursos de la carrera a través de experiencias pedagógicas en diversos contextos o escenarios educativos o sociales. Además, el plan de estudios contempla un curso de introducción a la investigación en el Bachillerato de la carrera y dos cursos en la licenciatura.

Para Hernández et ál. (2014) la investigación es "un conjunto de procesos sistemáticos, críticos y empíricos que se aplican al estudio de un fenómeno o problema" ( $p$ 4). En el marco disciplinar de la Orientación, se acuñan conceptos que integran la complejidad de este campo de estudio, con la visión integral del ser humano y todos sus procesos; se busca amalgamar en la formación académica, la producción del conocimiento y dominio metodológico, con la aplicabilidad en la práctica profesional, generando actitudes y aptitudes de las personas orientadoras-investigadoras. 
Hoy, los cambios sociales, políticos, geográficos, culturales y económicos llevan a plantearse nuevas formas de investigar dentro de la disciplina, y se visualiza como un reto contante el dominio metodológico de los paradigmas y enfoques en la aplicabilidad de la praxis disciplinar y el compromiso social en el ejercicio profesional. Se plantea como objetivo, potenciar desde la academia, la formación de profesionales en Orientación que esbocen propuestas exitosas sobre cómo enfrentar esos cambios a partir de un proceso investigativo y una sistematización rigurosa, además de fomentar profesionales que transmitan la pasión, el compromiso afectivo, ético y racional con el quehacer investigador.

\section{Rasgos de la mentalidad científica}

Giroux y Tremblay (2004) plantean cuatro rasgos que distinguen la mentalidad de un buen científico o científica, en el marco de la investigación de las ciencias humanas: la duda, la voluntad, la observación y la neutralidad mentalidad.

La duda se establece como el espacio y tiempo al que se acoge la persona investigadora para analizar un criterio y no emitir un juicio determinado o plantear una afirmación aun cuando parezca ser evidente.

La voluntad como rasgo científico plantea la capacidad de realizar observaciones asociadas a la medición, para así evitar subjetividades.

Cabe aclarar que no existe neutralidad en el proceso observación, ni en los lenguajes de descripción, sino intersubjetividades relativas. Najmanovich (citando a Maturana, 2008) menciona "'el camino explicativo de la objetividad entre paréntesis', que coloca al observador en el espacio de responsabilidad con respecto a lo que dice" (p.194).

El tercer rasgo que mencionan Giroux y Tremblay (2004) es la observación exhaustiva, como la capacidad de reunir todas las observaciones para establecer una relación lógica de investigación; y la cuarta característica es la neutralidad de mentalidad y la capacidad de tomar en consideración todas las observaciones y criterios, para así establecer principios teóricos.

\section{La investigación aplicada}

La investigación aplicada tiene la finalidad de generar conocimiento con la interacción directa en la sociedad, con lo cual existe una influencia en la vida de las personas, hechos o fenómenos sociales, educativos entre otros. Por tanto, "Lo propio de la investigación aplicada es estudiar problemas concretos con objeto de proponer un 'plan de acción' para decidir o intervenir eficazmente en una situación dada" (Giroux y Tremblay, 2004, p. 38). 


\section{La investigación en el campo disciplinar}

La investigación en la Orientación es parte del quehacer cotidiano profesional y repercute en las diversas acciones, servicios, estrategias y modelos de intervención, así como en las áreas educativas, vocacionales, profesionales y humanas. Por ende, dentro de la formación académica, se justifica no solo por su importancia en la vida profesional laboral, sino la necesidad de una formación rigurosa y consistente en este eje curricular y la disposición o actitud positiva hacia dicha práctica. Muñoz et ál. (2005) mencionan como resultado de sus experiencias docentes universitarias algunos puntos que justifican el aprendizaje de la investigación y destacan:

- La necesidad de formar un nuevo maestro capaz de hacer de la educación una práctica social de calidad.

- La necesidad de desarrollar investigación educativa para producir teoría pedagógica desde la práctica.

- La falta de comunidades académicas para pensar la educación por la vía del descubrimiento, la indagación, la reflexión, la acción, la escritura del saber pedagógico y la crítica. La necesidad de un modelo unificador que caracterice la actuación y el oficio de los egresados de las Unidades Formadoras de Educadores.

- La formación científica de talentos en el campo de la investigación, para que desde la educación básica inicien una motivación temprana sin tener que esperar hasta los tardíos niveles de posgrado....

- La utopía de intervenir la compleja realidad educativa, sin la cual no es posible el progreso de los pueblos. (p.16)

Estos principios aplicables a esta disciplina justifican y contextualizan la necesidad de formación en investigación y su vigencia. Las casas formadoras acuñan la responsabilidad de fomentar procesos pedagógicos que potencien las habilidades y destrezas en esta área, el desarrollo de nuevas teorías en contextos innovadores y prácticas profesionales sustentadas en estudios rigurosos con metodologías consistentes.

La docencia universitaria tiene la responsabilidad y urgencia de potenciar y maximizar los recursos humanos cualificados para satisfacer las necesidades educativas en investigación y fomentar dichos principios como elementos fundamentales del quehacer profesional.

\section{Investigación en el plan de estudios de Orientación}

El plan deestudios del año2006 dela carrera de Orientación dela Universidad Nacional (UNA, CIDE, DET, 2006) evidencia la importancia de la investigación en la formación de profesionales, se refuerza dicha acción desde los cursos específicos de la materia, la transversalización en la malla 
curricular, programas, proyectos o actividades académicas universitarias, así como trabajos finales de graduación y otros esfuerzos generados en diferentes gestiones administrativas de la División de Educación para el Trabajo, unidad académica a la cual pertenece la carrera de Orientación.

Dentro del plan de estudios, para el desarrollo profesional metodológico se cuenta con tres cursos de investigación, de cuatro créditos: uno en el plan de estudios de bachillerato y dos cursos en el plan de licenciatura; además, se enriquece las áreas disciplinares del desarrollo conceptualcontextual y personal social; esto es posible al ser la investigación un eje curricular, el cual se comprende como: "el proceso de observación, análisis y reflexión-acción-reflexión, constituye el segundo eje curricular de esta licenciatura y permite sustentar el proceso de orientación a partir de la realidad, la teoría y la práctica" (UNA, CIDE, DET, 2006, p. 26).

Adicionalmente, el estudiantado de esta carrera recibe el acompañamiento de una persona docente tutora durante la elaboración de los trabajos finales de graduación, quien se encarga de brindar asesoramiento metodológico a la población estudiantil durante su proceso de elaboración de tesis o proyecto, según sea la opción que escoja el estudiante.

Muñoz et ál. (2005), citando a Schon, 1998) afirman que:

los mejores profesionales son aquellos que van más allá de lo que pueden expresar con palabras. No basan sus conclusiones en fórmulas preconcebidas para afrontar su trabajo, sino en ese tipo de supervisión que sólo se aprende en la práctica. (p. 27)

De ahí, la trascendencia de la investigación en la Orientación, de la formación sólida y permanente de Orientares-investigadores.

Botero (2006, p. 55) plantea la importancia de los ejes transversales que permiten "dotar a los estudiantes de las capacidades necesarias para conocer e interpretar la realidad y poder actuar sobre ella". En este sentido, se recalca que, mediante la investigación, se puede conocer a fondo los diferentes fenómenos a los que se enfrenta la población estudiantil, y producto de ese conocimiento, se pueden realizar propuestas para interactuar de acuerdo con procesos basados en la sistematización de datos en contexto particular.

\section{Metodología}

El estudio investigativo se desarrolló desde un enfoque cuantitativo con alcance exploratorio, con el objetivo de indagar las características que debe desarrollar una persona profesional de Orientación, en los procesos de investigación, a partir del análisis de las fortalezas, debilidades, oportunidades y amenazas percibidas por el colectivo estudiantil en la formación académica de este eje curricular. 
El estudio se realizó en un lapso de cuatro años: 2014, 2015, 2016 y 2017, con una población de 15 estudiantes del curso Metodología de la investigación, en la licenciatura en Orientación; lo que permite visualizar en una línea de tiempo los resultados y las percepciones que se mantienen en relación con el tema. Esto brinda mayor profundidad y sustento a los resultados y su posterior análisis, además, los resultados son la base de varios encuentros académicos de discusión y reflexión en torno al tema de formación universitaria en investigación.

El estudio gira en torno a tres categorías de análisis, a saber: percepción de la formación académica en investigación, características de una persona orientadora-investigadora y aplicabilidad de la investigación en el quehacer profesional. Para recoger los datos en la primera categoría de análisis, se realizó un FODA, para las otras categorías se estableció un cuestionario con preguntas abiertas y semi-estructuradas. Se aplicó un pilotaje al cuestionario, en un grupo de 10 estudiantes de IV nivel del año 2014; posterior a esto, se realizó una validación por personas expertas y se contó con la revisión de un orientador profesor de cursos de metodología de la investigación.

En la semana 15 o 16 del curso de Metodología de Investigación II, se aplicaron los instrumentos, el criterio principal fue que el estudiantado ya estaba finalizando el curso y conocía mejor los procesos generales de la Universidad. Una vez recogida la información y sistematizados los resultados, se establecieron reuniones para revisar y analizar los procesos de formación investigativa universitaria.

\section{Resultados, análisis y discusión}

Los resultados se obtienen a partir de los instrumentos aplicados; sin embargo, la información se enriquece con las experiencias en docencia, la participación en Comisión de Trabajos Finales de Graduación, tutorías de trabajos finales de graduación, estudios investigativos interdisplinarios, y la constante reflexión y diálogo sobre los procesos de investigación con estudiantes.

\section{Percepción de la formación académica en investigación}

La información de esta categoría de análisis se recoge por medio de un FODA; además, se discuten algunas de las respuestas brindadas por el colectivo estudiantil con un grupo de personas académicas de la carrera de Orientación.

Los resultados corresponden a los principales hallazgos durante los cuatro años y los argumentos que coinciden en cada una de las categorías sobre debilidades, fortalezas, oportunidades, amenazas y posibles soluciones respecto a la formación académica en investigación según el colectivo estudiantil. 


\section{Debilidades:}

- Falta de objetividad en los análisis.

- Contradicción o divergencia en los puntos de vista del cuerpo docente y el equipo de investigación.

- Falta de recursos económicos.

- Dificultad en la redacción y ortografía de los procesos de investigación.

- Poco énfasis en la formación sobre procesos cualitativos.

- Poca validez del cuerpo teórico del documento.

- Falta de procesos vivenciales.

- El tiempo de las clases es limitado y resta espacio para producir y dar seguimiento de los procesos, lo cual afecta la calidad del producto.

\section{Fortalezas}

- Permite el análisis de temáticas emergentes.

- Claridad de la metodología de investigación por parte de los profesores del curso de investigación.

- Adquisición de conocimiento y espacios para la innovación.

- Acompañamiento del cuerpo docente.

- Abundancia de información para los trabajos de investigación, acceso a escenarios físicos de trabajo.

- Aprendizaje del proceso investigativo desde los primeros niveles.

- Coherencia en las regulaciones APA.

- Consolidación de grupos de trabajo.

- Formación en las etapas etarias del ser humano.

- Paralelismo entre el curso de investigación y los trabajos finales de graduación (TFG).

\section{Amenazas:}

- Cambios en los nombramientos de los profesores, la carga académica es variante.

- Divergencia entre profesores y comisión TFG.

- Estrés producto de la carga académica.

- Insuficiencia de tiempo. 
- La viabilidad de la población de investigación, una vez iniciado el proceso de TFG

- Desconocimiento del quehacer y roles de los profesionales en orientación por parte de otros profesionales.

- No divulgar los hallazgos de las investigaciones en otros escenarios profesionales en Orientación.

- Abundancia de trámites administrativos.

\section{Oportunidades:}

- Posicionamiento de la persona profesional y la disciplina de la orientación.

- Acceso a bibliografía.

- Los TFG podría generar apertura de nuevos escenarios de acción profesional.

- Profundizar en temas de interés y nuevos para la orientación.

- Construcción de conocimiento.

- Indagar en poblaciones específicas o nuevas.

- Crecimiento profesional y personal.

- Impacto social.

- Apertura de instituciones gubernamentales y no gubernamentales para investigar.

- instancias universitarias dedicadas a la investigación.

\section{Soluciones:}

- Promover la investigación aplicada a la sociedad.

- Procesos vivenciales practico-teórico.

- Generar proceso de seguimiento con la población.

- Proponer temas desde la CTFG.

- Agilizar procesos institucionales.

- Mejorar la maya curricular en licenciatura con una base práctica en investigación.

- Aumentar número de cursos de investigación en el bachillerato.

- Creación de un sistema de evaluación más integral y acorde a la realidad social nacional e internacional.

- Apertura para que la población estudiantil pueda conocer los TFG y publicar los resultados de las investigaciones. 
El FODA recoge los criterios del colectivo estudiantil relacionados con la formación investigativa en los últimos cuatro años en la Unidad Académica. Es importante destacar que estos son los puntos que más se mencionaron, son coincidentes y algunas debilidades se corrigieron a lo largo del tiempo.

El estudiantado de licenciatura en Orientación menciona como debilidades, las incongruencias existentes entreel personal docenteinvolucrado en los procesos deinvestigación, tanto de cursos como en la comisión de Trabajos Finales de Graduación, circunstancia que se ve reflejada en la poca claridad en los otros niveles del plan de bachillerato.

Se considera que imparten el curso sobre este tema tienen inferencia en dichos procesos, eximiendo al resto del cuerpo docente que imparte otros cursos; sin embargo, la investigación es una acción sustantiva que permea toda la malla curricular y debe estar presente en los objetivos de cada curso. Este argumento plantea el reto de visualizar y explicitar los procesos investigativos en cada uno de los cursos del plan de estudios y articular cada proceso a una investigación macro.

Las incoherencias mencionadas en el párrafo supracitado, también se perciben como amenazas durante los estudios investigativos en el tanto se encuentran divergencias entre el cuerpo docente y la comisión de TFG. El tiempo limitado, la carga académica y el exceso de trámites administrativos se conjugan como una amenaza para la investigación que realiza el estudiantado. Estos factores generan niveles elevados de estrés y son altamente incidentes en los procesos de trabajos finales de graduación; los procesos investigativos no pueden circunscribirse solo a procesos técnicos- administrativos, como menciona González et ál. (2007):

En el contexto educativo puede considerarse como un encuentro entre personas, es una actividad ética que requiere de continua reflexión y cuestionamiento, para ello no puede reducirse a una actividad técnica, debido a la profundidad del proceso, en ella participan docente-alumno-comunidad, en la cual, el docente actúa con todo lo que él es como sujeto, es decir, su comportamiento está enmarcado en sus creencias, actitudes, costumbres y entorno. De igual manera el alumno recibe información y la procesa de acuerdo con sus experiencias, costumbres y entorno, por ello el proceso de aprendizaje es una situación incierta, única, cambiante, compleja y presenta conflicto de valores tanto en la definición de las metas como en la selección de los medios. (p. 281)

Además de las amenazas, también se encontraron fortalezas importantes de considerar en cualquier proceso de investigación. Los cursos sobre esta materia permiten el análisis de temáticas emergentes, lo que conlleva a nuevos espacios de reflexión en torno a la epistemología de la disciplina, la praxis disciplinar y la interrelación coherente con el proceso investigativo. Esto facilita la apertura de un escenario educativo diverso y desde el aprendizaje significativo, 
como bien menciona la teoría del aprendizaje significativo, el conocimiento verdadero nace cuando los nuevos contenidos tienen un significado a la luz de los saberes que el sujeto ya posee, es decir, los conocimientos adquiridos encajan de tal forma que se reconfiguran en un nuevo significado.

Otra de las fortalezas mencionadas es la claridad metodológica de la investigación, esto permite que el estudiantado plantee sus ideas y pueda metodológicamente construir un cuerpo de conocimientos de la disciplina a partir de su propuesta de tema, con la finalidad de construir un proceso de investigativo desde la Orientación; generando una gama de oportunidades a nivel personal y profesional.

Dentro del FODA como elemento emergente, destacaron propuestas alternativas que desde la academia y con voluntad administrativa, se pueden generar para mejorar la enseñanza de los procesos de investigación de profesionales de Orientación, a saber: metodologías diversas, diseños de investigación social, IAP (investigación acción participación), clarificar y explicitar el eje transversal de un estudio investigativo en toda la carrera a nivel de bachillerato y licenciatura, formación docente que responda a los nuevos retos planteados por el colectivo estudiantil, entre otras acciones.

En la Figura 1, se observan las características deseables que plantea el colectivo estudiantil para una persona orientadora en los procesos de investigación. En un periodo de cuatro años, sobresalen rasgos como la perseverancia, actitudes reflexivas y el manejo del método y la técnica en investigación, los cuales coinciden con los argumentos presentados en el FODA, en donde la abundancia de tramitología y la poca claridad en los procesos y el estrés generado por la carga académica provocan en el estudiantado la necesidad de fortalecer este tipo de habilidades y características.

Figura 1: Características de la persona orientadora-investigadora

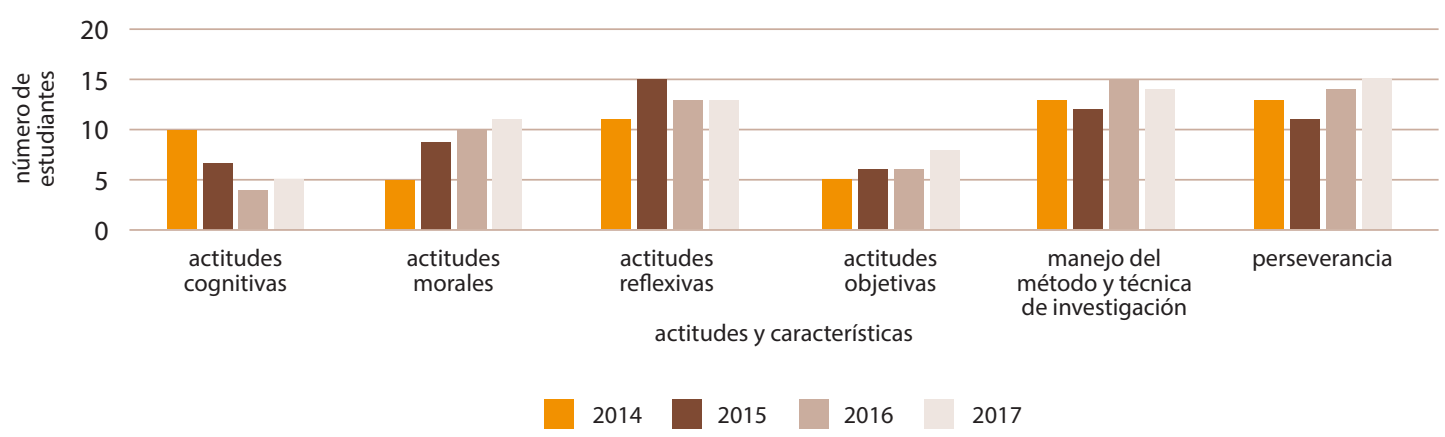

Nota: Elaboración propia. 
http://doi.org/10.15359/ree.24-2.13

ROR: https://ror.org/01t466c14 Universidad Nacional, Costa Rica

http://www.una.ac.cr/educare

educare@una.cr

Por tanto, se plantean actitudes que son fundamentales en la acción de investigar, y que la persona profesional en Orientación desarrolla a lo largo de la carrera, pues le permiten enfrentar los procesos de trabajos finales de graduación. La percepción estudiantil es que estas habilidades se convierten en un conjunto de características que todo profesional en orientación debe desarrollar.

El reto que se presenta es trabajar la integración de dichas fortalezas y actitudes en una simbiosis propia de la persona orientadora-investigadora, que permita que dichas actitudes se perpetúen a lo largo de toda su carrera profesional.

Algunas de estas características se presentan en la Figura 2 y son las más citadas por el grupo de estudiantes de la licenciatura de Orientación en los cursos de investigación.

Figura 2: Actitudes hacia la investigación en la disciplina de Orientación

\begin{tabular}{|l|}
\hline Actitud cognitiva \\
\hline - Manejo teórico \\
- Conocimiento estadístico \\
del tema a nivel nacional \\
e internacional \\
- Manejo de las \\
tecnologías \\
-Manejo de los idiomas \\
- Buen lector, apertura \\
\hline
\end{tabular}
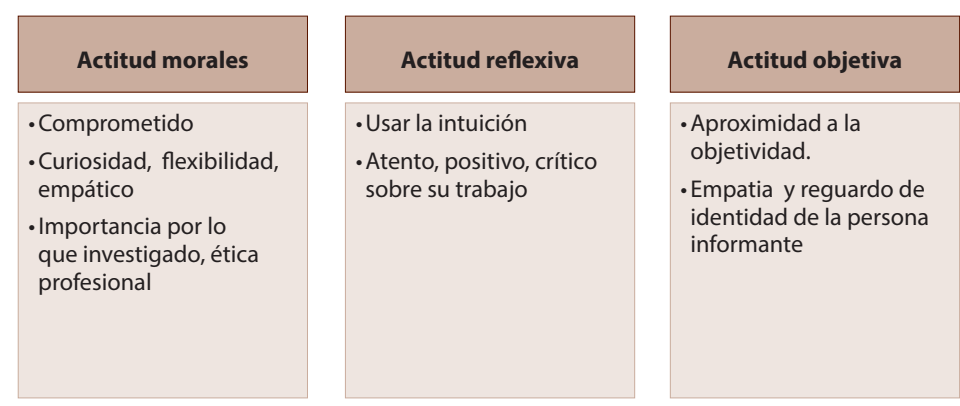

\begin{tabular}{|c|}
\hline $\begin{array}{l}\text { Manejo del metodo y } \\
\text { técnica de investigación }\end{array}$ \\
\hline - Fundamento teórico \\
\hline $\begin{array}{l}\text { - Constante actualización } \\
\text { teórica }\end{array}$ \\
\hline $\begin{array}{l}\text {-Capacidad de } \\
\text { interpretación }\end{array}$ \\
\hline - Disponibilidad de tiempo \\
\hline $\begin{array}{l}\text { - Habilidades para realizar } \\
\text { sistematización }\end{array}$ \\
\hline
\end{tabular}

Nota: Elaboración propia.

Las actitudes mencionadas por el colectivo estudiantil reafirman la necesidad de potenciar capacidades, habilidades positivas hacia la investigación desde el inicio de la carrera, y que estas se visualicen como básicas en el desarrollo profesional - personal y social del colectivo estudiantil.

\section{Importancia de la Investigación en Orientación}

En los últimos 4 años durante los procesos educativos llevados a cabo en los cursos de investigación en la carrera de Licenciatura en Orientación, se destacan elementos importantes para considerar en la formación universitaria y la repercusión de dicho análisis en los planes de estudio en cuanto a la investigación como eje transversal en toda la formación. Desde la percepción del colectivo estudiantil la formación rigurosa y el acompañamiento empático en los procesos metodológicos de investigación son fundamentales para poder ampliar conocimientos, mejorar las prácticas en orientación y abrir espacios académicos que permitan generar sinergias positivas en relación a la investigación.

\footnotetext{
12 Cindy Vanessa Artavia-Aguilar y Luis Roberto Campos-Hernández
}

Los artículos de la Revista Electrónica Educare del Centro de Investigación y Docencia en Educación de la Universidad Nacional, Costa Rica, se comparten bajo términos de la Licencia Creative Commons: Reconocimiento, № Comercial, Sin Obra Derivada 3.0 Costa Rica. Las autorizaciones adicionales a las aquí delimitadas se pueden obtener en el correo: educare@una.cr 
Considerando la investigación como un espacio de acción para construir, crear, y consolidar procesos propios de Orientación, el estudiantado a lo largo del tiempo manifestó algunos criterios que sustentan su importancia en la carrera.

Como se observa en la Figura 3, la investigación permite a la persona profesional en Orientación construir material didáctico, consolidar procesos de intervención con mejores resultados para el orientando, desarrollar nuevas habilidades y destrezas para intervención, la consolidación profesional, entre otros argumentos.

Figura 3: La importancia de la investigación en la disciplina de la Orientación

\begin{tabular}{|l|}
\hline \multicolumn{1}{|c|}{2014} \\
\hline - Ampliar los \\
conocimientos, mejores \\
abordaje en nuevos temas \\
- consolidación del \\
profesional en ORX, \\
apertura de nuevos \\
escenarios de acción \\
profesional \\
- creación de instrumentos, \\
producción realizada \\
en un lenguaje de la \\
disciplina \\
-variedad de temas a \\
investigar \\
-innovar en temas actuales \\
desde un abordaje \\
nacional e internacional \\
- permite conocer el \\
funcionamiento de las \\
dimensiones del ser \\
humano \\
- genera la fundamentación \\
teórica para nuevos \\
espacios no tradicion \\
\hline
\end{tabular}
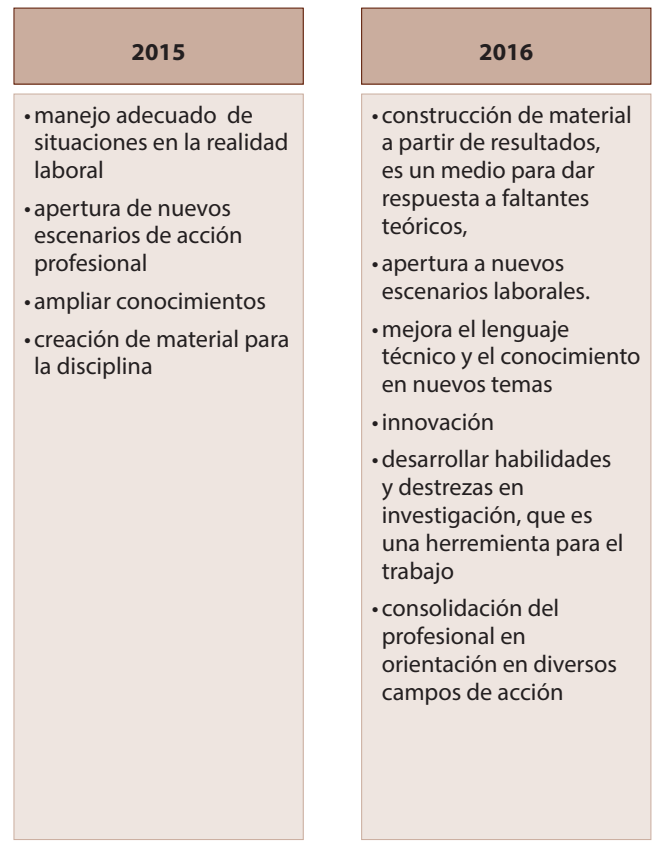

\begin{tabular}{|l|}
\hline \multicolumn{1}{|c|}{2017} \\
\hline - mejor conocimiento \\
del medio en que se \\
desarrolla el profesional \\
de la orientación \\
- se amplian \\
conocimientos teoricos y \\
prácticos \\
-innovación en el campo \\
disciplinar \\
- desarrollo de \\
habilidades y destrezas \\
en investigación para \\
nuevos escenario \\
laborales. \\
- construcción de \\
material, instrumentos, \\
y fundamentos teoricos \\
a partir de la reliadad \\
laboral.
\end{tabular}

Nota: Elaboración propia.

\section{Aplicabilidad de la investigación en el quehacer profesional de la orientación.}

Se considera que la investigación es un pilar fundamental en el quehacer profesional de la disciplina, en diferentes ámbitos de acción y en las modalidades de atención como: la atención individual, grupal, en los ciclos de la vida del ser humano, orientación vocacional, profesional y laboral. Como se observa en la Figura 4, el colectivo estudiantil considera que este eje curricular tiene criterios de aplicabilidad en diferentes áreas de desarrollo académico y laboral. 
Figura 4: Aplicabilidad de la investigación en el quehacer profesional de Orientación

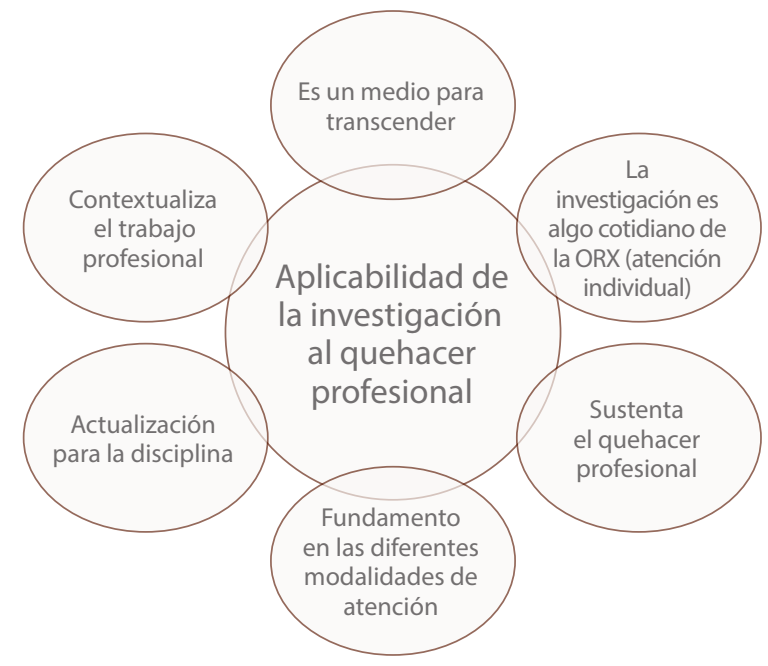

Nota: Elaboración propia.

La investigación sistemática e integrada a la cotidianidad de la labor profesional permite al individuo la actualización en diferentes temas y ámbitos de la disciplina, así como una atención contextualizada y coherente con las realidades del sujeto y su entorno sociodemográfico; lo que se podría reflejar en la realización de la persona profesional de la Orientación, Asencio et ál. (2015) plantea como nuevos retos de la investigación en cualquier campo relacionado a la educación, lo siguiente:

Entre los nuevos retos que hay que afrontar, que surgen, como hemos dicho, de los parámetros que definen la sociedad actual y que exigen enfoques novedosos, se encuentran múltiples ejemplos. Por citar algunos, nombraremos el desafío que plantea la educación de alumnos con necesidades educativas especiales y la atención educativa a la diversidad (Pumares Puertas y Hernández Rincón, 2010) o la revolución tecnológica y la incorporación de las TIC a los procesos de enseñanza-aprendizaje o el requerimiento de que el aprendizaje se extienda a lo largo de la vida (Long LifeLearning, LLL) (LópezZayas Barajas, 2006) y el aprendizaje auto-regulado (Self-RegulatedLearning, SRL). (p. 220)

\section{Conclusiones y recomendaciones}

La trascendencia de la investigación en la vida profesional de la persona Orientadora, y la integralidad de estos procesos en todo el quehacer profesional y de formación académica se sustenta con los resultados de este trabajo. 
La Orientación siendo una disciplina aplicada en las ciencias sociales, acompañado de un proceso de intervención entre dos o más personas según sea el caso y es un proceso de desarrollo personal y vocacional a lo largo de la vida, cimentado en el autoconocimiento, en el conocimiento de las condiciones, oportunidades, características y perspectivas que ofrece el medio y en la adquisición de destrezas para tomar decisiones, aprendizajes que las personas necesitan para planificar su vida. (Pereira, 2004, p. 7)

Se considera fundamental dentro de los procesos de desarrollo personal, social y educativo, la exploración de diferentes contextos en los que se desenvuelve el ser humano; por tanto, la persona orientadora tiene la responsabilidad de formarse en metodología de la investigación para un desempeño óptimo de su quehacer profesional, que se encuentre sustentando en conocimientos científicos, actualizados y acordes a la realizad del contexto en que se desenvuelve.

Una persona orientadora-investigadora en ciencias humanas fortalece lo que Giroux y Tremblay (2004) plantean para ser un buen científico, a saber: la duda, la voluntad, la observación y la neutralidad mentalidad; considerando siempre las intersubjetividades relativas.

Como principales hallazgos se pueden retomar que la investigación, vista como un proceso en la formación y en la vida profesional, requiere de ajustes constantes a la realidad nacional e internacional plasmados en programas de cursos, orientaciones pedagógicas y curriculares, líneas de investigación, relación y coherencia metodológica en todos los años de estudio de la carrera; pero, sobre todo, coherencia entre los estamentos universitarios.

Se concluye, además, la necesidad de conducir los procesos hacia una formación académica de la Orientación de calidad, comprometida con la responsabilidad social, lo que lleva a promover el desarrollo de la investigación educativa para producir nuevos conocimientos, postulados teóricos contextualizados a la realidad social, nacional y global; en donde la formación académica tenga: "diferentes acciones interestructuradas e intencionalizadas que puedan insinuar caminos posibles de actuación por donde se direccione dicho proceso" (Alzate, 2015, p.14).

Los procesos de investigación (cursos, trabajos finales de graduación, entre otros) deben ser empáticos, con metodologías de indagación, reflexión constante, acción y análisis de nuestras propias prácticas de orientación, que conlleven a un modelo que caracterice el actuar y los fundamentos teóricos del estudiantado durante su proceso formativo y posterior a este, con motivación constante al quehacer investigativo; así mismo, se visualiza la importancia de sintonizar por parte de los académicos y académicas, la forma en que se lleva a cabo la investigación y el establecimiento de lineamientos congruentes y homologados en la Carrera de Orientación para elaborar los diferentes reportes.

En la misma línea, la comunidad académica docente requiere de espacios de diálogo reflexivos y de acción sobre el trabajo investigativo en todos los procesos de orientación. 


\section{Referencias}

Alzate, F. A. (2015). Prácticas y formación docente: Un escenario propicio para promover la investigación educativa en Colombia. Actualidades Investigativas en Educación, 15(2), 1-17. https://doi.org/10.15517/aie.v15i2.18962

Asencio, I. I., Ruiz, C. y Castro, M. (2015). Formación de maestros e investigación educativa: La percepcióndelosestudiantes degradoenlauniversidadComplutensedeMadrid.Tendencias Pedagógicas, 26, 217-236. https://dialnet.unirioja.es/servlet/articulo?codigo $=5247173$

Botero, C. A. (2006). Los ejes transversales como instrumento pedagógico para la formación de Valores. Revista Politécnica, 2(3), 49-59. http://revistas.elpoli.edu.co/index.php/pol/article/ view $/ 58$

Giroux, S. y Tremblay, G. (2004). Metodología de las ciencias humanas. La investigación en acción. Fondo de Cultura Económica.

González, N., Zerpa, M. L., Gutiérrez, D. y Pirela, C. (2007). La investigación educativa en el hacer docente. Laurus, 13(23), 279-309. http://www.redalyc.org/articulo.oa?id=76102315

Hernández, R., Fernández, C. y Baptista, P. (2014). Metodología de la investigación (6ª ed.). McGraw Hill.

Muñoz, J. F., Quintero, J. y Munévar, R. A. (2005). Cómo desarrollar competencias investigativas en educación (3. ${ }^{\mathrm{a}}$ ed.). Cooperativa Editorial Magisterio.

Najmanovich, D. (2008). Mirar con nuevos ojos. Nuevos paradigmas en la ciencia y pensamiento complejo. Editorial Biblos.

Pereira, T. (2004). Orientación educativa. EUNED.

Universidad Nacional, Centro de Investigación y Docencia en Educación, División Educación para el Trabajo. (2006). Rediseño del plan de estudios: Bachillerato y licenciatura en orientación. Autor. 